\title{
Kinematic Distances of SNRs W44 and 3C 391
}

\author{
Hongquan $\mathrm{Su}^{1}$, Wenwu Tian ${ }^{1}$, Hui $\mathrm{Zhu}^{1} \&$ F. Y. Xiang ${ }^{2}$ \\ ${ }^{1}$ National Astronomical Observatories, Chinese Academy of Sciences, \\ 100012, Beijing, China. email: hq_su@bao.ac.cn \\ ${ }^{2}$ Dept. of Physics, Xiangtan University, 411105, Xiangtan, Hunan Province, China
}

\begin{abstract}
We extracted the neutral hydrogen absorption spectra of supernova remnants W44 (G34.7-0.4) and 3C 391 (G31.9+0.0) from the VLA Galactic Plane Survey data. The revised distance of W41 is about $3.3 \mathrm{kpc}$ replacing the previous $3.0 \mathrm{kpc}$. Further, we confined the distance of G31.9+0.0 to about $7.2 \mathrm{kpc}$ due to its interaction with its surrounding molecular clouds.
\end{abstract}

Keywords. methods: data analysis, techniques: radial velocities, ISM: supernova remnants

\section{Introduction}

Distance of supernova remnants (SNRs) is not only a basic parameter of finding their properties such as radius and explosion energy, but also a key factor to research the related high energy phenomena. Recently, the kinematic distances of more than 10 SNRs have been revised by analyzing their $\mathrm{H}$ I absorption spectra and ${ }^{13} \mathrm{CO}$ spectra (Tian \& Leahy 2011). Here we report our measurement on SNRs W44 and 3C391 which are related with high energy phenomena and interacting with their surrounding environment. Our measurements are based on the assumption of the basic Galactic parameters $\mathrm{V}_{\odot}=$ $220 \mathrm{~km} \mathrm{~s}^{-1}, \mathrm{R}_{\odot}=8.5 \mathrm{kpc}$.

\section{W44}

W44 (also named G34.7-0.4, 3C392) has a shell-like radio-continuum morphology (Figure 1.) with a centrally peaked distribution of thermal X-ray surface brightness (Cox et al. 1999). It lies in a complex region which is close to several molecular clouds and a giant molecular cloud (GMC) complex (Dame et al. 1986).

We have obtained its H I absorption spectrum (Figure 1.) from the VLA Galactic Plane Survey (VGPS) data. This spectrum shows the highest absorption velocity at $50 \mathrm{~km} \mathrm{~s}^{-1}$ much less than the velocity of tangent point in the direction of W44. This gives a lower limit distance of $3.3 \mathrm{kpc}$ for W44. The H I spectra of W44 show H I emission but no associated absorption beyond the velocity of $\sim 50 \mathrm{~km} \mathrm{~s}^{-1}$. This constraints W44 at a distance of $3.3 \mathrm{kpc}$. Caswell et al. (1975) found the highest velocity absorption feature of $42 \mathrm{~km} \mathrm{~s}^{-1}$ then concluded that the distance of W44 was $3 \mathrm{kpc}\left(\mathrm{R}_{\odot} \sim 10 \mathrm{kpc}\right.$ was adopted) with low velocity resolution data. The VGPS observations provide the strongest evidence to date that the W44 is no further than $3.3 \mathrm{kpc}$ distant; in turn, this supports earlier conclusions that the $\mathrm{OH}(1720 \mathrm{MHz})$ maser emission, which peaks at a velocity of $46.9 \mathrm{~km} \mathrm{~s}^{-1}$ (Claussen et al. 1999), is physically associated with the W44. 

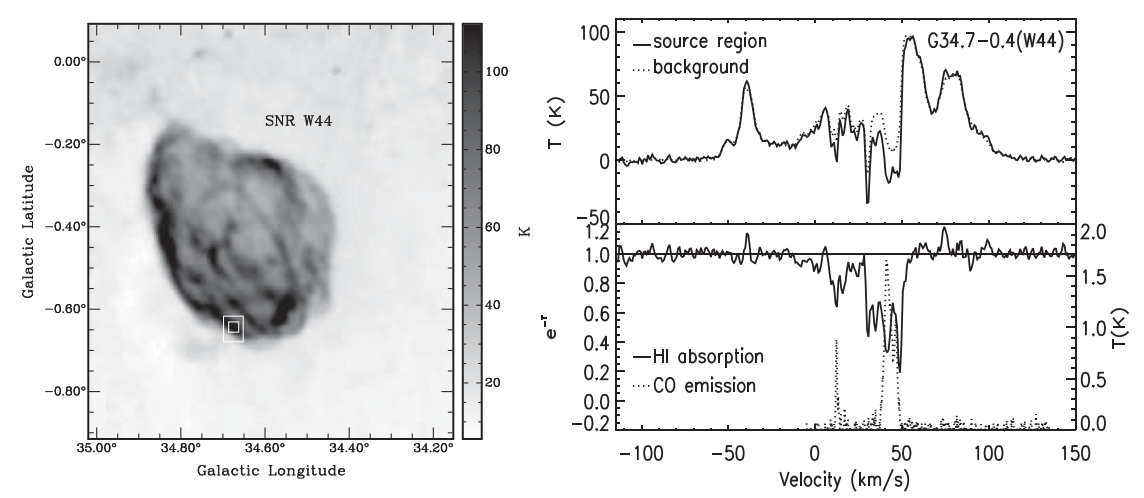

Figure 1. The $1420 \mathrm{MHz}$ continuum image of W44 (left). The H I and CO emission apectra and H I absorption spectrum of W44 (right).

\section{3. $3 \mathrm{C} 391$}

3C 391 (also named G31.9+0.0) is a thermal composite remnant, with centerbrightened thermal X-ray emission (Rho \& Petre 1996). The radio morphology of 3C 391 is a half shell of radius $5^{\prime}$, with another half extend out the open end of the shell which suggests it breakouts into a region of significantly lower density (Renolds \& Moffett 1993).

New H I absorption spectrum of 3C 391 reveals the highest absorption velocity at 108 $\mathrm{km} \mathrm{s}^{-1}$, i.e. at tangent point, this implies a lower limit distance of $\sim 7.2 \mathrm{kpc}$ for $3 \mathrm{C} 391$. There is $\mathrm{H}$ I emission peak at $70 \mathrm{~km} \mathrm{~s}^{-1}$ but lack of absorption, which hints the $\mathrm{H}$ I cloud lies behind the continuum source 3C 391. So we give an upper limit distance of $10.4 \mathrm{kpc}$.

This conclusion is supported by several $\mathrm{H}$ I emission peaks without associated $\mathrm{H} \mathrm{I}$ absorptions in the velocity from 30 to $50 \mathrm{~km} \mathrm{~s}^{-1}$. Previous studies showed that this SNR has an interaction with clouds at the tangent point (Wilner et al. 1998). We conclude that $3 \mathrm{C} 391$ is most likely at a distance of $7.2 \pm 0.3 \mathrm{kpc}$. The uncertainty of $0.3 \mathrm{kpc}$ is from the Galactic parameter's uncertainty and the measurement errors.

Caswell et al. (1971) suggested a distance of $8.5-13.4 \mathrm{kpc}$ for 3C 391 because of the $\mathrm{H} \mathrm{I}$ absorption velocity at $105 \mathrm{~km} \mathrm{~s}^{-1}$ and the lack of absorption at $35-60 \mathrm{~km} \mathrm{~s}^{-1}$ (for a Galactocentric distance of $10 \mathrm{kpc}$ ) by Parkes $21 \mathrm{~cm}$ line observations.

\section{Acknowledgement}

We acknowledge supports from the NSFC program (011241001, 11261140641, 11273022). It is also supported by a grant of from the John Templeton Foundation and NAOC.

\section{References}

Caswell, J. L., Dulk, G. A., Goss, W. M., Radhakrishnan, V., \& Green, A. J. 1971, A\&A, 12, 271

Caswell, J. L., Murray, J. D., Roger, R. S., Cole, D. J., \& Cooke, D. J. 1975, A\&A, 45, 239

Claussen, M. J., Goss, W. M., Frail, D. A., \& Desai, K. 1999, ApJ, 522, 349

Cox, D. P., Shelton, R. L., Maciejewski, W., Smith, R. K., Plewa, T., Pawl, Andrew., \& Ryczka, M. 2011, ApJ, 524, 179

Dame, T. M., Elmegreen, B. G., Cohen, R. S., \& Thaddeus, P. 1986, ApJ, 305, 892

Reynolds, S. P. \& Moffett, D. A. 1993, AJ, 105, 2226

Rho, J.-H. \& Peter, R. 1996, ApJ, 467, 698

Tian, W. W. \& Leahy, D. A. 2011, ApJ, 729, L15

Wilner, D. J., Reynolds, S. P., \& Moffett, D. A. 1998, AJ, 115, 247 